\title{
Exstirpation eines Orbitaltumors mit Erllaltung des Bulbus
}

\author{
von \\ Dr. Wilh. Zehender, \\ Medicinal-Assessor in Neustrelitz.
}

Dis bisher bekannt gewordenen Fälle von glücklicher Exstirpation grosser Orbitaltumoren mit Erhaltung des Augapfels sind nicht so zahlreich, dass wir nicht hoffen dürften, die ausführliche Mittheilung cines solchen Falles werde von den Freunden des ophthalmologischen Archivs mit einigem Interesse gelesen werden.

Dergleichen Tumoren gehören überhaupt unter die selteneren Erkrankungen und zeigen übcrdies in ihrem Auftreten in ihrer Struktur, ihrer Grösse, ihrer Localisirung, ihrer Einwirkung auf den Augapfel und dessen Schvermügen mannigfache Verschiedenheiten.

In den meisten zur Behandlung kommenden Fällen ist das Auge bereits so weit zerstört oder erkrankt, dass an eine Erhaltung des Bulbus gar nicht mehr gedacht werden kann, oder man verzweifelt unter den gegebenen Verhältnissen an ein Gelingen der Erhaltung des Bulbus, oder endlich man versucht den Bulbus zu erhalten und die Erhaltung gelingt nicht. Unter den 
verhältnissmässig wenigen Fällen, in welchen die Erhaltung wirklich dauernd geglückt ist, finden wir über die Beschaffenheit des Bulbus und seines Sehvermögens vor und nach der Operation gar oft weniger gesagt, als wir zu wissen wünschen.

Dies hat uns veranlasst, den von uns beobachteten Fall einer öffentlichen Mittheilung nicht ganz unwerth zu halten.

Eine ziemliche Anzahl hierher gehöriger Fälle finden sich gesammelt in den grösseren Werken von Mackenzie, Tyrrel und Lawrence, wie auch in der.Monographie über Orbitaltumoren von D em arqua y.**)

Fälle von glücklich operirten (weder knöchernen noch flüssigen) Orbitaltumoren mit Erhaltung des Sehvermögens finden sich ausgeführt und beschrieben von St. Yves, ${ }^{* *}$ ) von Thomas Hope, ${ }^{* * *}$ ) von Barnes, $\dagger$ ) von Langenbeck, $t+$ ) von Dupuytren, $+t+$ ) Maisonneuve, $\left.{ }^{*}\right)$ Chassaignac, $\left.{ }^{* *}\right)$ ZachariahLaurence $e^{* * *}$ ) und Andere mehr.

Wir wollen hier die Kranken-Geschichte unseres Falles folgen lassen.

Gegen Ende October 1857 meldete sich bei mir

*) Des tumeurs de l'orbite. Thèse présentée et soutenue par $\mathbf{M}$. Demarquay. Paris 1853.

$\left.{ }^{* *}\right)$ Nouveau Traité des Maladies des Yeux. Paris 1722. Pag. 147.

***) Philosoph. Transact. 1744-45.

f) Medico-Chirurgical Transactions. London 1813. Vol. IV. Pag. 316.

†f) Neue Bibliothek für Chirurgie und Ophthalmologie. Hannover 1819. Bd. II. S. 238 und 40.

tft) Lancette française. 1835. Pag. 446.

*) Gazette des Hopitaux. 1841.

**) Demarquay des tumeurs de l'orbite. Pag. 172.

***) Medical Times and Gazette. N. 409. May 1858. Pag. 449. 
eine 44jährige Bauernfrau von dem Gute Boek in Mecklenburg-Schwerin mit Exophthalmos des linken Auges.

Die Frau hatte mehrere gesunde Kinder geboren und, ihrer Angabe nach, nie an irgend einer erheblichen Krankheit gelitten. Deber die Entstehung des Augenleidens wusste sie keine genügende Auskunft zu geben. Sie sagte, dass die ersten Anfänge sich schon vor mehr als einem Jahr, vielleicht schon vor zwei Jahren bemerklich gemacht hätten, dass aber erst seit Ostern 1857 das Uebel beträchtlich geworden und rascher als bisher zugenommen habe. Der dortige Gutsarzt Dr. Probsthan aus Mirow hatte sie bereits mit ableitenden und resolvirenden Mitteln: mit Einreibungen, Salben, Vesikantien u. s. w. behandelt, kurz hatte Alles versucht, was unter den gegebenen Verhältnissen Hülfe und Linderung zu versprechen schien. Doch war trotz dem der Exophthalmos unaufhaltsam fortgeschritten.

Als die Kranke sich mir vorstellte, konnte über das Vorhandensein eines Orbitaltumor kein $Z_{\text {weifel obwalten. }}$ In der Gegend des äusseren und oberen Orbitalrandes liess sich unter dem Augenlide eine weiche, etwas gelappte Geschwulst deutlich durchfühlen. Der Augapfel war stark nach vorn, jedoch nicht nach unten, sondern mehr nach oben gedrängt. Ein geringer Grad von Ptosis bedingte daher schon eine vollkommene Bedekkung der Cornea des protrudirten Bulbus. Wenn man das linke obere Augenlid durch einen Assistenten etwas emporziehen liess und nun von der rechten Seite her möglichst parallel zur Gesichtsfläche visirte, so erschien der Scheitelpunkt der rechten Cornea etwa 5 Lin. hinter, der Scheitelpunkt der rechten Cornea dagegen 9 Lin. vor dem Nasenrücken, so dass die Differenz der Entfernung beider Scheitelpunkte von einer zur Ge. sichtsfläche parallelen. Ebene beinabe fünfviertel Zoll betrug. Um so viel war also der. Bulbus des kranken 
Auges aus seiner normalen Lage verdrängt. Hiernach durfte wohl das Volumen des Tumor auf mehr als das doppelte Volumen des Bulbus geschätzt werden. Dieser Tumor hatte sich nun nach aussen und etwas nach unten an dem Bulbus vorbei gedrängt, so dass die Cornea - wie eben bemerkt wurde - unter dem oberen Augenlide versteckt lag. Nur durch gewaltsames Aufreissen, insbesondere unter Verschluss des rechten Auges, war es der Kranken möglich, die versteckte linke Cornea zu befreien, wobei sie versicherte, mit diesem Auge vollkommen gut sehen zu können. Das untere linke Augenlid war ektropionirt und durch eine kolossale fleischige Hypertrophie seiner Conjunctiva in dieser Lage dergestalt zurückgehalten, dass man nur mit einiger Gewalt eine Sonde zwischen das Lid und die Hautbedeckung der Wange hindurchführen konnte. Wir wollen hier noch bemerken, dass die angeblich seit Ostern eingetretene Verschlimmerung wahrscheinlicherweise auf die Entstehung und allmälige Vergrösserung dieses Ektropium zu beziehen sei.

Beim Emporziehen des oberen Augenlides zcigte der Augapfel eine geringfügige centrale Hornhauttrübung; im Uebrigen aber nichts Krankhaftes. Die Sehschärfe hatte verhältnissmässig wenig gelitten, doch war durch den von hinten nach vorn wirkenden Druck der Geschwulst ein hoher Grad von Hyperpresbyopie hervorgerufen. Die Kranke sah mit Convex 9 recht gut in der Ferne und las kleine Druckschrift (Schrift VI der Jäger'schen Probelettern) in ciner Entfernung von 6 bis 8 Zoll mit Convex 4.

Das Allgemeinbefinden der Kranken war gut, doch war ihr Gemüth in hohem Grade deprimirt. Sie versicherte, in letzterer Zeit vergesslich geworden zu sein und Nachts nur sehr unruhig, oft auch gar nicht geschlafen zu haben, doch will sie weder in der Tiefe der 
Augenhöhle noch überhaupt im Kopfe jemals erhebliche Schmerzen empfunden haben.

Nur die zu Tage liegende Conjunctiva des unteren Augenlides, welche sie mit einem trockenen Leinwandläppchen zu bedecken pflegte, verursachte ihr oberflächliche Schmerzen.

\section{Operation.}

Wie schlecht auch die Prognose bei Orbitalgeschwülsten im Allgemeinen sein mag, so schien sie doch wegen der Abwesenheit aller Schmerzen und wegen der Unversehrtheit des Bulbus und seines Sehvermögens in diesem Falle wenigstens relativ gut gestellt werden zu können.

Am 7. November unternahm ich daher den Versuch einer Exstirpation der Geschwulst mit Erhaltung des Augapfels, unterstützt von meinen hiesigen Collegen, den Herren DD. Götz und Eggers.

Ich verlängerte zunächst die Augenlidspalte nach aussen bis einige Linien über den Orbitalrand hinaus, so dass die ganze Länge des Schnittes vielleicht ${ }_{4}^{3}$ Zoll betrug, liess die Augenlider sowohl wie die Wundränder möglichst von einander abziehen und suchte nun, mehr mit stumpfen Instrumenten und mit dem Finger, als durch den Schnitt wirkend, in die Tiefe der Augenhöhle zu dringen. Die seitliche Loslösung der Geschwulst gelang ziemlich leicht, dagegen haftete sie in der Tiefe und zwar in der Nachbarsehaft der fissura Spheno-maxillaris und an dem planum orbitale alae magnae ossis Sphenoidei besonders fest, so dass es nur mit Mühe und mit Anwendung einiger Gewalt gelang, sie von diesen Stellen gänzlich loszutrennen. Die Consistenz der Geschwulst war überdies nicht beträchtlich genug, um die Entfernung in einer einzigen Masse zu gestatten, vielmehr zerdrückte und zertrümmerte sie sich, sobald man 
genöthigt war, eine etwas grössere Gewalt zu ihrer Lostrennung anzuwenden. Dennoch konnte sie bis auf einigen Detritus, welcher nachträglich so sorgfältig als möglich entfernt wurde, in zwei Hauptportionen weggenommen werden.

Der Blutverlust bei dieser Operation war unbedeutend und die Kranke fühlte sich hauptsächlich nur durch die Chloroformnarkose, welche ein mehrmaliges Erbrechen zur Folge hatte, belästigt.

$\mathrm{Nach}$ behandlung.

Nach Vollendung der Operation wurde der Augapfel nicht in die entstandene Lücke reponirt, diese Lücke wurde vielmehr durch vorsichtiges Ausstopfen mit Charpie noch zugängig erhalten und über das Ganze ein leichter Compressivverband gelegt, welchen wir erst nach Ablauf von zweimal 24 Stunden in derselben Weise erneuerten.

Da der untersuchende Finger noch einige nicht ganz unverdächtige Stellen fand, so wurde eine concentrirte Aetzpaste von Chlorzink ganz dünn auf Leder gestrichen und hiervon ein fünfviertel Zoll langer und zwei Lin. breiter Streifen mit der ätzenden Fläche nach aussen, bis nahe an den, für den untersuchenden Finger leicht fühlbaren Sehnerven eingeführt; der freie Raum zwischen der Rückenfläche des Lederstreifchens und dem Bulbus nobst seinem Sehnerven möglichst schützend durch Charpie ausgefüllt. Die Wirkung dieser Aetzpaste war sehr ausgiebig. Sie zerstörte nicht nur alle noch etwa verdächtigen Stellen, sie entblösste sogar noch den knöchernen Rand der Orbita, während nach innen die Charpie kaum hinreichte, den Sehnerven und Augapfel zu schützen; denn die Aussenfläche des Augapfels überzog sich sogar noch mit einem ganz dünnen Brandschorf, welcher indessen die Sclerotica unversehrt liess. 
Am vierten Tage nach Einführung der Aetzpaste konnte der Schorf, wiewohl nicht ganz ohne Gewalt, in Form einer konischen Röhre herausgezogen werden, worauf sich die von anscheinend ganz gesunder Wundfläche ausgekleidete Lakune zeigte. Dieselbe wurde jetzt abermals und von nun an alltäglich mit Charpie einigermassen fest ausgefüllt und über das Ganze ein leichter Compressivverband angelegt; im Uebrigen aber jeder Versuch vermieden, den Bulbus in seine normale Lage zurück zu drängen.

Der Kranken wurden innerlich nur unbedeutende, dem Allgemeinbefinden entsprechende Arzneimittel verabreicht.

Uebrigens war ihr Befinden bei einer so schmerzhaften und eingreifenden Cur ziemlich befriedigend. Symptome, die zu ernster Besorgniss Veranlassung hätten geben können, waren nicht vorhanden.

Unter der eingeschlagenen Behandlung zog sich der Bulbus allmälig ganz von selbst in seine richtige Lage zurück, so dass nach Ablauf von drei Wochen die Lakune bereits so verengt und verkleinert war, dass man kaum mit der Spitze des kleinen Fingers noch oberflächlich eindringen konnte und dass ein Unterschied in der Lage der beiden Bulbi durch die oben angegebene Untersuchungsmethode nicht mehr nachweisbar blieb.

Das Ektropium des unteren Augenlides, welches bei der Operation ganz unberücksichtigt blieb, wurde nach einigen Tagen mit mitigirtem Höllenstein leicht touchirt. Es verkleinerte sich dergestalt, dass es am 10. oder 12. Tage durch den Verband schon vollkommen reponirbar war und nach Ablauf von drei Wochen nur noch als eine kleine, wiewohl etwas derbe Conjunctivalfalte sichtbar blieb.

Der Augapfel hatte seine Beweglichkeit zwar nicht 
verloren, aber doch einen ziemlich beträchtlichen Theil derselben eingebüsst. Die Pupille, welche in den ersten Tagen nach der Operation ad maximum erweitert und vollkommen unbeweglich war, zeigte erst am 13. oder 14. Tage bei sehr genauer Beobachtung ganz minime Schwankungen auf Lichtreiz. Diese Schwankungen wurden von dieser Zeit an immer leichter und deutlicher wahrnehmbar, doch ist dic Pupillenweite nicht ganz wieder zur Norm zurückgekehrt. Die centrale Hornhauttrübung verschwand gänzlich.

Ein genaueres Examen über die Beweglichkeit des Bulbus, über die Grösse und Beweglichkeit der Pupille und über die Sehschärfe wurde erst nach Verlauf mehrerer Wochen angestellt. Einestheils schien die Zeit, in welcher hierin allmälige Veränderungen eintraten, kein besonderes Interesse zu verdienen, anderntheils war es nicht wohl thunlich, die Kranke länger als nöthig zu tagtäglicher Beobachtung am hiesigen Orte zurückzuhalten. - Nachdem sie daber_wieder fähig war, ihre häuslichen Arbeiten zu verrichten, d. h. nach einem Zeitraum von acht Wochen, wurde sie entlassen, unter der Bedingung, sich anfangs von acht zu acht Tagen, später aber in längeren $Z$ wischenräumen wieder zu präsentiren.

Der Gemüthszustand der Kranken hatte sich auffallend gebessert. Früher hatte sie sich wegen des ekelerregenden Anblicks seit 3 Jahren nur mit verbundenem linken Auge unter Menschen gewagt und war durch die Furcht vor dem immer zunehmenden Uebel auf's Aeusserste beunruhigt und geängstigt. Gegenwärtig ist sie heiter und gesellig und achtet die zurückgebliebenen Unbequemlichkeiten fast gar nicht.

Beschaffenheit des Tumor.

Das Gewicht des exstirpirten und von Blutcoagulis 
gereinigten Tumor betrug 5 Drachmen, doch darf dasselbe wohl noch etwas höher veranschlagt werden, da die bei der nachträglichen Reinigung der Wunde entternten letzten kleinen Trümmer nicht mitgewogen wurden. Die Grösse konnte wohl auf das zwei- bis drittehalbmalige Volumen des Bulbus geschätzt werden. Die Oberfläche war uneben drusig, die jüngsten und oberflächlichsten Partien weich, die tieferen dagegen und vornehmlich diejenigen, an denen der Tumor an den Orbitalwandungen fest sass, consistenter und fast knorpelig fibrös. Die Elemente der jüngsten Schichten waren rundliche oder mehr ovale Kerne, von denen einige schon schwache Andeutungen einer umhüllenden Zellenmembran zeigten. In den tieferen Schichten aber fanden sich neben den genannten Elementen zahlreiche Kernfasern und spindelförmige Zellen, welche die Tendenz zur Umwandlung in Bindegewebsbildungen nicht wohl verkennen liessen. Es war dies demnach ein gutartiger fibro-plastischer Tumor.

Die Beweglichkeit des Augapfels.

Da die Kranke anfangs durch Doppelbilder belästigt wurde, so konnte der Grad der Beweglichkeitsstörung ziemlich gut bemessen werden. In späterer Zeit verlor sich die Fähigkeit Doppelbilder zu sehen immer mehr und mehr, auch strebten wir danach diese Störung durch ein blaues Planglas möglichst zu beseitigen. Dennoch konnten bis in die jüngste Zeit durch ein vor das rechte Auge gehaltenes dunkelrothes Glas die Doppelbilder sehr leicht wieder hervorgerufen werden.

Neun Wochen nach der Operation stellte ich die ersten genaueren Beweglichkeits-Prüfungen, welche, geringe Schwankungen abgerechnet, so wenig von den letzten (sechs Monate nach der Operation angestellten) Prüfungs-Ergebnissen verschieden sind, dass eine Bes- 
serung seit jener Zeit kaum angenommen werden darf. Wir theilen deshalb nur dasjenige Prüfungsresultat mit, welches zwischen den einzelnen Schwankungen am besten die Mitte hält.

Allgemeines Prüfungsresultat.

In Folge der zerstörenden Einwirkung der Operation einestheils, und in Folge narbiger Zusammenziehungen und Verwachsungen anderntheils, war an dem kranken Auge zurückgeblieben: eine Insufficienz der mm. recti ext. int. und sup., während der rect. inf. keine Anomalie seiner Function zeigte. Die Function der obliqui blieb unbestimmbar, weil die Kranke mit Bezug auf die Schrägstellung der Doppelbilder keine zuverlässigen Angaben machte. Bald läugnete sie die Schrägstellung ganz, bald machte sie darüber ungenügende oder widersprechende Aussagen.

Prüfung der Maximalwirkung der Augenmuskeln.

Um die Maximal-Wirkung der vier graden Augenmuskel zu ermitteln, wurde die Kranke 12 Zoll vor eine weisse, zur Gesichtsfläche möglichst parallele Wand gestellt und angewiesen einen im Niveau der Augenlidspalten und gerade vor ibr befindlichen Punkt zu fixiren. Alsdann wurde ihr aufgegeben, bei verschlossenem rechten Auge und unveränderter Kopfstellung ein successive nach rechts, nach links und nach oben gehaltenes Object zu fixiren. Die Grenze, an welcher die Möglichkeit der direkten Fixation aufhörte und das excentrische Sehen begann, wurde angemerkt. Diese Grenze bezeichnete uns die Grenze der grösstmöglichen Muskelwirkung.

Der am 14. Mai 1858 angestellte Versuch ergab nach der unmittelbaren Distanzmessung von dem an- 
fänglichen Fixationspunkt und der daraus berechneten ungefähren Winkelgrösse folgendes Resultat:

nach oben 4 Zoll oder $18^{\circ}$,

"rechts $15, \quad, 50^{\circ}$,

$"$ links $13, " 45^{\circ}$.

Nach links konnte das Auge noch etwas weiter, wiewobl nicht ganz bis in den inneren Augenwinkel gewendet werden, der Nasenrücken verhinderte aber die genauere Bestimmung dieser Grenze. - Nach unten war die Grenze der Beweglichkeit wie auf dem gesunden Auge,

Prüfung der associirten Augenbewegungen.

Die Kranke hielt vor das gesunde rechte Auge ein dunkelrothes Glas, wodurch die Doppelbilder leicht zur Erscheinung gebracht werden konnten. Mit einem weissen Stäbchen, welches gleichfalls doppelt erschien, wurde sie angewiesen, den Ort anzugeben, wo sie die Spitze eines vorgehaltenen Objektes (gewöhnlich ein brennendes Licht) sah, so zwar, dass das weisse Bild des Stäbchens auf das rothe Bild der Kerzenflamme wies. Hierdurch konnte der Ort des excentrischen Bildes mit Bezug auf seine Höhe, seine Entfernung und seine seitliche Abweichung ziemlich genau ermittelt werden. Dieser Ort ist aber kein absolut genauer, denn er ist den Schwankungen der Muskelinnervation unterworfen, und es dürfen daher geringe Schwankungen in den Angaben noch nicht als Fehler der Beobachtung angesehen werden.

Die Prüfung der associirten Augrenbewegungen geschah in einer Entfernung von 12 Zoll und zwar so, dass

1. das Objekt in der Medianlinie bei aufrechter Kopfhaltung und in der Höhe der Augenlidspalten gehalten wurde. Dann wurde

2. das Objekt in einer zur Gesichtsfläche möglichst Archiv für Ophthaimologie. IV. 2. 
parallelen Ebene, deren Entfernung gleichfalls 12 Zoll betrug, zunächst nach links bewegt; (von der Kranken aus gerechnet) bis an die Grenze, in welcher die Doppolbilder nicht mehr gesehen werden konnten, d. h. in welcher das rechte Auge durch den Nasenrücken verhindert war, das Objekt noch zu sehen. Dieser Grenzpunkt fand sich in einer Richtung, welche mit der Medianlinie einen Winkel von etwa $45^{\circ}$ einschloss. Dann wurde

3. das Objekt in derselben Weise nach rechts bewegt und endlich

4. in derselben Weise, d. h. gleichfalls um etwa 45 nach oben und

5. nach unten gehalten.

Die Prïfung dieser fünf Augenstellungen ergab nach verschicdenen Versuchen folgendes Verhältniss:

1. Das Bild des kranken Auges steht in derselben Vertikale, aber 4 Zoll höher als das andere.

2. Das Bild des kranken Auges steht 7 Zoll weiter nach links, 4-5 Zoll höher und etwas näher. Nach einer späteren Prüfung betrug der seitliche Abstand nur 5 Zoll, die Höhendifferenz 4 Zoll.

3. Das Bild des kranken Auges steht 5 bis 6 Zoll weiter nach rechts und 8 Zoll höher und etwas näher. Nach einer späteren Prüfung 4 bis 5 Zoll weiter nach rechts und 9 Zoll höher. Das Gebiet des Geradeübercinanderstehens beider Bilder erstreckt sich von der Medianlinie aus etwas nach rechts.

4. Das Bild des kranken Auges steht um 8 Zoll höher, fällt aber in dieselbe Vertikale.

5. Beide Bilder stehen gleich hoch und in derselben Vertikale, ohne jedoch zu einem einzigen - wenn ich so sagen darf - stereoskopischen Bild zusammen zu fallen.

Wir dürfen aus diesen Daten wohl kanm schliessen, 
dass eine wesentliche Besserung in den Motilitätsverhältnissen der Muskeln statt gefunden habe.

Prüfung der accommodativen Augenbewegungen.

In der Medianlinie blieben die Doppelbilder in allen Entfernungen des Objekts, trotz der Höhendifferenz in derselben Vertikale.

Bei der ersten Prüfung (14. Januar) wurde in einer Entfernung von 6 Zoll gekreuztes Doppelsehen mit dem seitlichen Abstande einer kleinen Fingerbreite zugestanden; später aber sogar in einer Nähe von 3 oder 4. Zoll noch geläugnet.

\section{Die Papillenweite.}

Die Messung der scheinbaren Pupillenweite geschah mit einem möglichst nahe vor das Auge gehaltenen Cirkel und zwar in vertikaler Richtung. Wir stellen aus mehreren Versuchen, bei denen jedesmal wenigstens zwei Messungen gemacht wurden, folgende kleine Tabelle zusammen, in welcher die gefundenen Zahlen in Zollen nach Wiener Maass angegeben sind.

\begin{tabular}{c|c|c|c}
\hline $\begin{array}{c}\text { Datum } \\
\text { des Versuchs. }\end{array}$ & $\begin{array}{c}\text { Zahl der Wochen } \\
\text { nach der Operation. }\end{array}$ & Rechtes & Linkes \\
Auge.
\end{tabular}

Zu bemerken ist hierzu noch, dass der zweite Versuch (28. Jan.) an einem sehr hellen, durch Schneere- 
flex sogar blendenden Tage angestellt wurde und dass die linke Pupille überhaupt etwas lebhafter auf Lichtreiz zu reagiren schien, als die rechte.

\section{Die Sehschärfe.}

Acht Wochen nach der Operation (7. Januar) wurde die Sehschärfe zum erstenmal einer genaueren Prïfung unterworfen.

Die Kranke las von den Jäger'schen Probelettern: mit dem rechten Auge ohne Brille Schr. III

$$
\text { " " " } " \text { und Convex } 20 \text { Schr. I. }
$$

Die Kranke ist mithin auch auf dem rechten Auge etwas presbyopisch, worauf vor der Operation nicht geachtet worden war.

mit dem linken Auge, ohne Brille Schr. XV.

$$
" \text { " " und Convex 4. Schr. III., }
$$

fast ganz richtig und beinahe fliessend, nur - wie sie sich ausdrückte — „etwas schämmerig." Von Schr. II. einzelne Worte.

Die Prüfung des 16. März, mithin 17 Wochen nach der Operation ergab sogar mit dem linken Auge und Convex Schr. I.

mühsam, aber correct, mit Ausnahme der schwereren Worte. - Ohne Brille Schr. XVI., ebenso wie Schr. I mit Convex 4.

Da die Krânke mit Convex 4 vor der Operation nur Schr. VI. las, so hat sich mithin das Sehvermögen dieses Auges unzweifelhaft gebessertt eine Besserung, welche wir uns nicht gut anders, als durch das gänzliche Verschwinden der leichten centralen Hornhauttrübung zu erklären wussten.

Hiermit haben wir einen ziemlich ausführlichen und wie wir hoffen, auch ziemlich erschöptenden Bericht über den gegenwärtigen Zustand der Kranken gegeben, woraus zugleich ersichtlich ist, dass sich dieser Zustand 
in den letzten acht Monaten weder zum Bessern noch auch zum Schlechteren verändert habe. Obwohl dieser Zeitraum unsere anfänglichen Befürchtungen bereits wesentlich verringert hat, so müssen wir doch gestehn, dass ein Rest von Befürchtung vor zukünftigen Recidiven sich bei uns noch immer nicht ganz will bekämpfen lassen. Hierzu scheint uns ein etwas längerer Beobachtungs-Zeitraum durchaus erforderlich. Wir halten es für eine Pflicht, welcher wir uns nicht enthebon wollen - vorausgesetzt, dass wir die Kranke nicht ganz aus dem Gesicht verlieren - in der nächsten oder nachnächsten Lieferung des Archiv's eine kurze Notiz über das derzeitige Befinden der Kranken nachzutragen. 